\title{
Role of transient receptor potential channel 6 in the odontogenic differentiation of human dental pulp cells
}

\author{
XIAOTING YANG ${ }^{1,2^{*}}$, ZHI SONG $^{1,2^{*}}$, LINGLING CHEN $^{1,2}$, RUNFU WANG $^{1,2}$, \\ SHUHENG HUANG ${ }^{1,2}$, WEI QIN ${ }^{1,2}$, JIA GUO $^{1,2}$ and ZHENGMEI LIN ${ }^{1,2}$ \\ ${ }^{1}$ Department of Operative Dentistry and Endodontics; ${ }^{2}$ Guangdong Provincial Key Laboratory of Stomatology, \\ Guanghua School of Stomatology, Hospital of Stomatology, Sun Yat-sen University, \\ Guangzhou, Guangdong 510055, P.R. China
}

Received February 5, 2016; Accepted February 10, 2017

DOI: $10.3892 /$ etm.2017.4471

\begin{abstract}
Pulp capping is a restorative technique employed in an attempt to maintain pulpal vitality and generate reparative dentin. $\mathrm{Ca}^{2+}$ released from capping materials is suggested to promote reparative dentin formation. Transient receptor potential channel 6 (TRPC6) is a receptor-operated $\mathrm{Ca}^{2+}$ channel that serves an important role in $\mathrm{Ca}^{2+}$ influx in the majority of non-excitable cells, and influences the calcium signaling and cell respond. Therefore, the purpose of the present study was to gain an insight into the role of TRPC6 in the odontoblastic differentiation of human dental pulp cells (HDPCs). Human dental pulp tissues and HDPCs were obtained from healthy third molars. By immunohistochemical staining, TRPC6 was observed to be highly expressed in the dental pulp tissue, particularly in the odontoblast layer. In addition, the protein level of TRPC6 was increased in a time-dependent manner during odontogenic differentiation of HDPCs. Downregulation of TRPC6 by a lentivirus vector containing TRPC6 shRNA inhibited the process of odontogenic differentiation in HDPCs. In conclusion, the current data demonstrated that TRPC6 served a significant role in the odontogenic differentiation of HDPCs, suggesting it may be a promising therapeutic target in regenerative endodontics.
\end{abstract}

\section{Introduction}

The prime objective of restorative dentistry is to maintain pulpal vitality and function. Occasionally, pulp tissue may

Correspondence to: Dr Zhengmei Lin, Department of Operative Dentistry and Endodontics, Guanghua School of Stomatology, Hospital of Stomatology, Sun Yat-sen University, 74 Zhongshan Road, Guangzhou, Guangdong 510055, P.R. China

E-mail: linzhm@mail.sysu.edu.cn

${ }^{*}$ Contributed equally

Key words: transient receptor potential channel 6, odontoblasts, cell differentiation, calcium be exposed accidentally during a clinical procedure, such as deep caries removal or restorative procedures. In cases where the pulp tissue is healthy or diagnosed with reversible pulpitis, direct pulp capping is regarded as an effective approach to preserve the pulp vitality and function by forming a dentinal bridge against pulp exposure $(1,2)$. Calcium ions $\left(\mathrm{Ca}^{2+}\right)$ released from calcium hydroxide or mineral trioxide aggregate have been certified as classic direct pulp capping materials, and are key factors promoting the healing process (3-5). Besides, as a ubiquitous second messenger, $\mathrm{Ca}^{2+}$ is involved in abundant physiological functions, including proliferation and differentiation. The role of calcium signaling in osteogenic differentiation is of prime interest for both normal bone homeostasis and bone regeneration (6). However, the mechanism underlying the effect of $\mathrm{Ca}^{2+}$ on the odontoblastic differentiation of dental pulp cells has yet to be fully elucidated.

The elevation of cytosolic calcium concentration can be achieved by two major mechanisms: Discharge of intracellular $\mathrm{Ca}^{2+}$ stores, and extracellular $\mathrm{Ca}^{2+}$ influx. $\mathrm{Ca}^{2+}$ current is mediated mostly by calcium channels located in membranes. The intracellular stores are regulated by inositol triphosphate $\left(\mathrm{IP}_{3}\right)$ receptors, while extracellular $\mathrm{Ca}^{2+}$ influx is mainly regulated by the canonical transient receptor potential (TRPC) channels and the ORAI channels (7). ORAI channels and certain TRPC members are regulated by depletion of intracellular stores, which refers to the store-operated $\mathrm{Ca}^{2+}$ entry (SOCE) (8). SOCE is a broadly existent mechanism in non-excitable cells, responding to the cytosolic $\mathrm{Ca}^{2+}$ change and refilling $\mathrm{Ca}^{2+}$ stores. A recent study revealed that ORAI1 is involved in the odontogenic differentiation of human dental pulp cells (HDPCs), which implied that $\mathrm{Ca}^{2+}$ mobilization is vital for the odontogenic differentiation (9). Furthermore, several TRPC members are activated by diacylglycerol in the store-independent manner, which refers to the receptor-operated $\mathrm{Ca}^{2+}$ entry (ROCE) (8). Another previous study demonstrated that rat odontoblasts expressed RNA transcripts for TRPC1 and TRPC6 (10).

TRPC6, the $\mathrm{Ca}^{2+}$-permeable non-selective cation channel, has been suggested as a ROCE channel. Activation of TRPC6 by diacylglycerol (DAG) analogues, such as 1-oleoyl-2-acetyl-sn-glycerol (OAG) (11), resulted in elevated intracellular $\mathrm{Ca}^{2+}$, which then influenced the calcium entry or calcium signaling. However, limited information is available 
on the importance of TRPC channels in dental pulp cells. Consequently, the present study hypothesized that TRPC6 is expressed in the human dental pulp and participates in the odontogenic differentiation of dental pulp cells.

\section{Materials and methods}

Tooth preparation. In this study, all teeth were obtained from the Department of Oral and Maxillofacial Surgery, Guanghua School and Hospital of Stomatology, Sun Yat-sen University, (Guangzhou, China) between December 2014 and February 2015. A total of 22 caries-free human wisdom teeth were extracted from 22 different patients aged 18-25 undertaking orthodontic treatment. All patients provided informed consent prior to treatment. The experimental protocols were approved by the Ethics Committee of the Guanghua School of Stomatology.

Tissue sample preparation. Four healthy teeth were randomly sectioned to reveal the healthy pulp inside, fixed with $4 \%$ paraformaldehyde overnight and then decalcified in 10\% EDTA for 3 months. Subsequently, decalcified tissues were embedded in paraffin and placed onto slides. Following deparaffinization, five random sections with integral structure in each tooth were selected for immunohistochemical staining. Four pulp tissue samples from another four teeth were prepared to detect TRPC6 for western blotting, as described below.

Cell culture and odontoblastic differentiation. HDPCs were established from healthy third molars by growing the minced explants as described previously (12). In brief, teeth were split and the dental pulp tissue was collected into a $35-\mathrm{mm}$ Petri dish. The tissues were minced into pieces and cultured antiseptically during the whole process. Cells were cultured in Dulbecco's modified Eagle's medium (DMEM) with 10\% fetal bovine serum (FBS) at $37^{\circ} \mathrm{C}$ in the presence of $95 \%$ air and $5 \%$ $\mathrm{CO}_{2}$. To induce odontogenic differentiation, cells at passage 3 were grown in 6 -well dishes at a density of $1 \times 10^{5}$ cells per well with a DMEM containing $10 \% \mathrm{FBS}, 0.2 \mathrm{mmol} / \mathrm{l}$ ascorbic acid, $10 \mathrm{mmol} / 1 \beta$-glycerophosphate and $100 \mathrm{nmol} / \mathrm{l}$ dexamethasone. The medium were refreshed every 2 days for 14 days. Subsequently, Alizarin red S staining was performed to confirm the mineralization. HDPCs from four healthy third molars extracted from patients (two female and two male) were used to detect TRPC6 expression level by western blotting analysis. In addition, HDPCs from another 10 teeth were prepared for the subsequent experiments, such as the experiment to induce odontoblast differentiation of HDPCs, $\mathrm{Ca}^{2+}$ concentration detection and the knockdown experiments.

The human embryonic kidney 293T cell line was purchased from American Type Culture Collection (Manassas, VA, USA). 293T cells were grown in $100 \mathrm{~mm}^{2}$ dishes at a density of $1.2 \times 10^{6}$ cells per well with DMEM containing $10 \%$ FBS.

Short hairpin RNA (shRNA) and lentivirus constructs. Lentivirus-based shRNA knockdown pLKO.1 puro vector (Addgene plasmid \#8453; Addgene, Cambridge, MA, USA) was used to stably knockdown the expression of TRPC6 (Genbank accession no. NM_004621.5), and shRNA constructs were generated as described previously (13). Target sequences (Thermo Fisher Scientific, Inc., Waltham, MA, USA) were selected through software provided on the manufacturer's website. Oligo sequences were annealed, subcloned into the pLKO.1 vector by T4 DNA ligase between the AgeI and EcoRI sites, according to the manufacturer's protocols. The selected TRPC6 hairpin oligo sequence for the study was as follows: 5'-GCGACAGAGCATCATTGACGCAAATCT GTGAAGCCACAGATGGGATTTGCGTCAATGATGCTC TGGTGCTTTTTT-3'. In addition, a non-target scrambled shRNA (with a sequence of 5'-GCGCGTAGTAATGACAAT CCGCGCTCTGTGAAGCCACAGATGGGAGCGCGGATT GTCATTACTACTTGCTTTTTT-3') served as the negative control. The shRNA-expressing vectors were transfected into 293T cells together with the lentiviral helper plasmids to generate respective lentiviruses. After $48 \mathrm{~h}$, lentiviruses were collected from the culture medium and used to infect target cells. HDPCs were transfected with the lentiviruses three times a day, and the medium was replaced with growth medium supplemented with $10 \%$ FBS. Next, medium containing $2 \mu \mathrm{g} / \mathrm{ml}$ puromycin (Sigma-Aldrich; Merck KGaA, Darmstadt, Germany) was used to isolate cells stably transduced with shRNA. The successful knockdown of TRPC6 was verified by western blot analysis.

Western blot analysis. Total protein was extracted from the HDPCs or pulp tissue samples using RIPA medium containing phenylmethanesulfonyl fluoride (Beyotime Institute of Biotechnology, Haimen, China). Protein concentrations were determined using a BCA Protein Assay kit (P0012; Beyotime Institute of Biotechnology). Equivalent amounts of diluted protein samples were separated by $10 \%$ sodium dodecyl sulfate-polyacrylamide gel electrophoresis and transferred onto a polyvinylidene difluoride membrane. After blocking for $1 \mathrm{~h}$ at room temperature in 5\% skim milk solution, the membranes were subsequently incubated with anti-TRPC6 antibodies (1:500; ab62461; Abcam, Cambridge, UK), anti-dentin sialophosphoprotein (anti-DSPP) antibodies (1:500; sc73632; Santa Cruz Biotechnologies, Inc., Dallas, TX, USA), anti-dentin matrix protein-1 (anti-DMP-1) antibodies (1:500; sc73633; Santa Cruz Biotechnology, Inc.) overnight at $4^{\circ} \mathrm{C}$. Secondary antibody anti-mouse IgG/anti-rabbit IgG, HRP-linked antibody (7076S/7074S; Cell Signaling Technology, Inc., Danvers, MA, USA) was then added at a dilution of 1:5,000 for $1 \mathrm{~h}$ at room temperature after the membranes were washed with Tris-buffered saline with Tween-20. Relative band intensities were detected by densitometry using Quantity One 1-D analysis software (version 4.6.2; Bio-Rad Laboratories, Inc., Hercules, CA, USA).

Immunohistochemical staining. Immunohistochemical staining was performed mainly in the humidity chamber as described in a previous study (12). Briefly, each section was pretreated with $3 \%$ hydrogen peroxide in ice-cold methanol for $20 \mathrm{~min}$ in order to block endogenous peroxidase activity. Next, slide-loaded sections were bathed in $0.01 \mathrm{M}$ sodium citrate buffer ( $\mathrm{pH}$ 6.0) at $95-100^{\circ} \mathrm{C}$ in a microwave oven for antigen retrieval. After blocking with normal goat serum for $20 \mathrm{~min}$ at room temperature, slides were incubated with primary anti-TRPC6 antibody (1:100; ab62461; Abcam) overnight at $4^{\circ} \mathrm{C}$. Slides were subsequently incubated with the secondary 
antibodies, goat anti-rabbit IgG (1:200; ab205718, Abcam) and stained with 3,3'-diaminobenzidine (Boster Systems, Inc., Pleasanton, CA, USA) and hematoxylin according to the manufacturer's instructions. Finally, the slides were observed using a light microscope.

Measurement of cytosolic $\mathrm{Ca}^{2+}$ concentration $\left(\left[\mathrm{Ca}^{2+}\right]_{c}\right)$. HDPCs at passage 3 were plated into 96-well assay plates at a density of $1 \times 10^{5}$ cells per well and loaded with $5 \mathrm{mM}$ Fura 2/AM for $30 \mathrm{~min}$ at $37^{\circ} \mathrm{C}$. $\left[\mathrm{Ca}^{2+}\right]_{\mathrm{c}}$ in cells was determined by a spectrofluorometer at excitation wavelengths of 340 and $380 \mathrm{~nm}$, and an emission wavelength of $510 \mathrm{~nm}$. Background fluorescence intensities were subtracted from each data point. The calcium concentration can be calculated according to the following formula: $\left[\mathrm{Ca}^{2+}\right]_{c}=\mathrm{K}_{\mathrm{d}} \mathrm{x} \beta \mathrm{x}\left(\mathrm{R}-\mathrm{R}_{\min }\right) /\left(\mathrm{R}_{\max }-\mathrm{R}\right)$, where $K_{d}$ represents the dissociation constant and $\beta$ is a constant. $\mathrm{R}_{\max }$ and $\mathrm{R}_{\min }$ were obtained while the cells were perfused and exposed to high and zero calcium settings, respectively (14). Next, $100 \mu \mathrm{M}$ OAG (Sigma-Aldrich; Merck KGaA) was used as an analogue of DAG to activate TRPC6. The absence of extracellular $\mathrm{Ca}^{2+}$ was determined by incubation of HDPCs in $\mathrm{Ca}^{2+}$-free Hank's balanced salt solution (Thermo Fisher Scientific, Inc.) with $3 \mathrm{mM}$ EGTA. Supplement of extracellular $\mathrm{Ca}^{2+}$ was achieved by addition of $2 \mathrm{mmol} / \mathrm{l} \mathrm{CaCl}_{2}$.

Statistical analysis. The values are demonstrated as the mean \pm standard deviation. Statistical analyses were performed using one-way analysis of variance, followed by the Bonferroni multiple-comparison test, with a statistically significant difference detected when $\mathrm{P}<0.05$. Statistical analyses were performed with SPSS 17.0 (SPSS Inc., Chicago, IL, USA).

\section{Results}

Expression of TRPC6 in human dental pulp tissue and HDPCs. Pulp tissue harbors various cell types. To determine which type of cells in the pulp tissue express TRPC6, the TRPC6 antibody was used to perform immunohistochemical staining in the pulp tissue samples. The results indicated that TRPC6 was expressed in the dental pulp tissue particularly in the odontoblast layer (Fig. 1A). In the negative control group, no positive staining was detected in the healthy dental pulp (Fig. 1A). In addition, the protein expression of TRPC6 in pulp tissue, as well as in HDPCs, was further detected by western blotting. TRPC6 protein expression in dental pulp tissues was significantly higher compared with that in HDPCs ( $\mathrm{P}<0.05$; Fig. 1B and $\mathrm{C})$. Thus, these findings suggest that both human dental pulp tissues and HDPCs expressed TRPC6.

TRPC6 is markedly upregulated during odontoblastic differentiation of HDPCs. Subsequent to confirming that TRPC6 is expressed in HDPCs by immunohistochemical staining, the expression levels of TRPC6, as well as that of the odontoblastic differentiation-associated proteins DSPP and DMP-1, were further analyzed during cell differentiation. In the differentiated cells, the expression level of TRPC6 was enhanced in a time-dependent manner compared with that in undifferentiated cells, with significantly increased levels observed at 7 and 14 days ( $\mathrm{P}<0.05$; Fig. $2 \mathrm{~A}$ and $\mathrm{B})$. A similar trend was observed for the DSPP and DMP-1 expression levels $(\mathrm{P}<0.05$; Fig. 2A, $\mathrm{C}$ and $\mathrm{D})$.

Knockdown of TRPC6 inhibits the odontoblastic differentiation of HDPCs. To verify whether the upregulation of TRPC6 was indispensable during odontoblastic differentiation, lentiviruses contained TRPC6 shRNA were used to knock down TRPC6 expression in HDPCs. The results demonstrated that the lentiviruses expressing shRNA were able to knock down the endogenous TRPC6 by up to 75\% (Fig. 3A). In addition, TRPC6 knockdown functionally inhibited $\mathrm{Ca}^{2+}$ influx, indicating that ROCE was impaired (Fig. 3B). In the TRPC6-shRNA group, a significant decline was detected in the calcium nodule formation (Fig. 3C). Accordingly, the protein levels of DSPP and DMP-1 were all also reduced in the TRPC6 knockdown groups on day 14 after odontoblastic induction (Fig. 3D and E), which indicated that knockdown of TRPC6 inhibited the odontoblastic differentiation of HDPCs.

\section{Discussion}

TRPC6 is a receptor-operated $\mathrm{Ca}^{2+}$ channel that serves an important role in regulating $\mathrm{Ca}^{2+}$ influx in the majority of non-excitable cells (15-17). A recent study reported that TRPC6 is expressed in the brain, kidney, smooth muscle tissues, as well as in immune and blood cells (18). In the present study, it was demonstrated that TRPC6 was expressed in dental pulp tissue, and was required for the odontogenic differentiation of HDPCs. To the best of our knowledge, this is the first study reporting an essential role of TRPC6 in the odontoblastic differentiation of HDPCs.

Although a recent study demonstrated that TRPC6 mRNA was detected in rat odontoblasts by single-cell reverse transcription-polymerase chain reaction (10), there is limited knowledge about the role of TRPC6 in dental pulp cells. The results of the present immunohistochemistry experiments revealed that TRPC6 was expressed in human dental pulp tissue and mainly in the odontoblast layer. Odontoblasts are considered to be the terminally differentiated cells, which are responsible for dentin formation. During dentin formation, $\mathrm{Ca}^{2+}$ is conveyed to the extracellular mineralization front against concentration gradients by odontoblasts (19). In contrast to odontoblasts, HDPCs can be easily obtained from extracted teeth. Besides, HDPCs as a heterogeneous population consist of the multipotent stem/progenitor cells that can differentiate into odontoblast-like cells during the formation of the reparative dentin due to the similar phenotypical properties they share with odontoblasts $(20,21)$. Since the endogenous expression of TRPC6 in HDPCs was detected by western blotting in the present study, it is presumed that TRPC6 was involved in the odontoblastic differentiation of HDPCs.

In vitro experiments in the present study identified that TRPC6 expression was significantly enhanced in a time-dependent manner when odontoblastic differentiation was induced in HDPCs, particularly on days 7 and 14 . This finding leads to the question of whether TRPC6 was indispensable during odontoblastic differentiation. Thus, the function of TRPC6 in the odontoblastic differentiation of HDPCs was explored. The results demonstrated that 
A
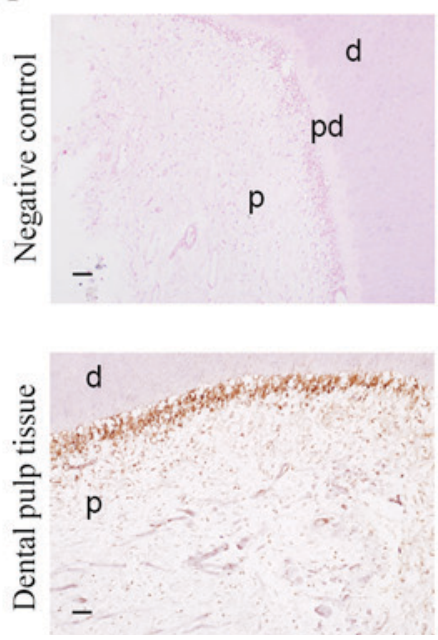
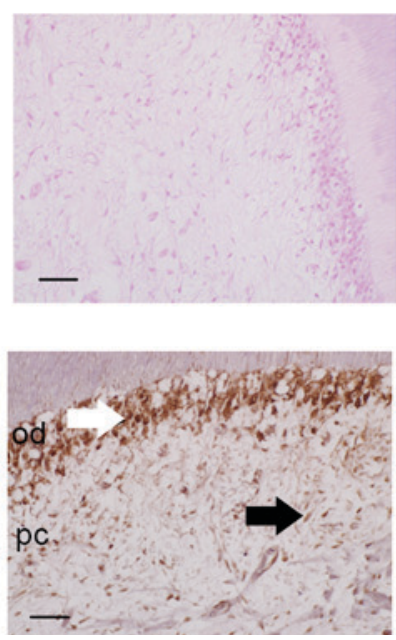

B

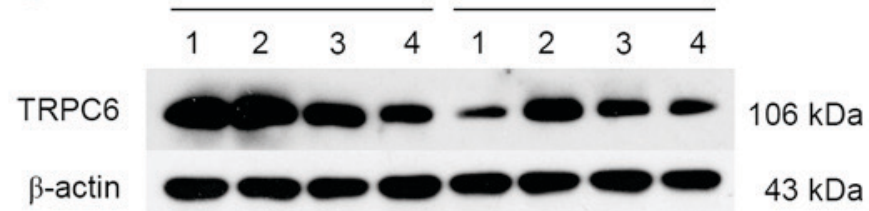

$\mathrm{C}$

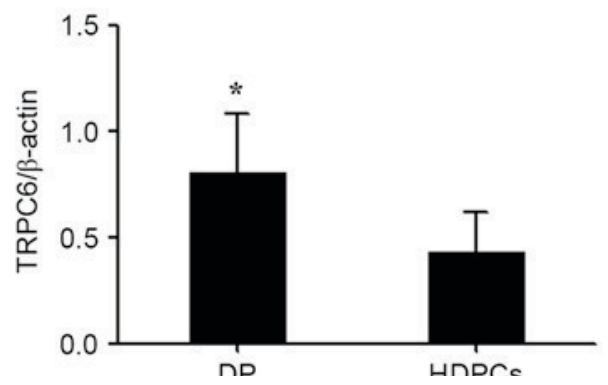

Figure 1. Expression of TRPC6 in human dental pulp tissue and HDPCs. (A) Positive anti-TRPC6 staining was detected in the cytoplasm of odontoblasts (white arrow) situated in the outermost layer of healthy dental pulp. Positive anti-TRPC6 staining was not evident in the pulp fibrobasts (black arrow) and the negative control of healthy pulp tissues. Scale bar, $100 \mu \mathrm{m}$. Upper images, magnification x200. Lower images, magnification x400. (B) Expression of TRPC6 protein $(106 \mathrm{kDa})$ was detected by western blotting in the dental pulp tissue $(\mathrm{n}=4)$ and HDPCs $(\mathrm{n}=4)$. $\beta$-actin was used as a loading control. (C) Densitometric values for each group are shown in the bar graph as the mean \pm standard deviation. All data are representative of three separate experiments. "P<0.05 vs. HDPCs. HDPCs, human dental pulp cells; TRPC6, transient receptor potential channel 6; DP, dental pulp; d, dentin; pd, predentin; p, pulp; od, odontoblast; pc, human dental pulp cell.

A

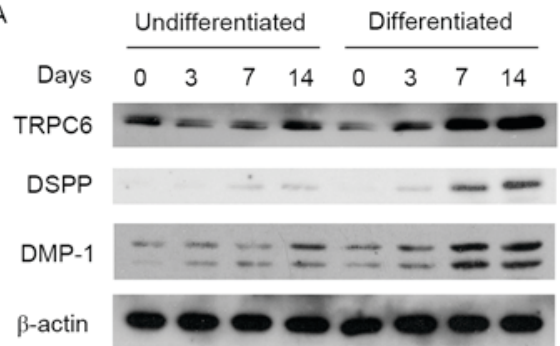

$106 \mathrm{kDa}$

$101 \mathrm{kDa}$

$57 \mathrm{kDa}$

$43 \mathrm{kDa}$

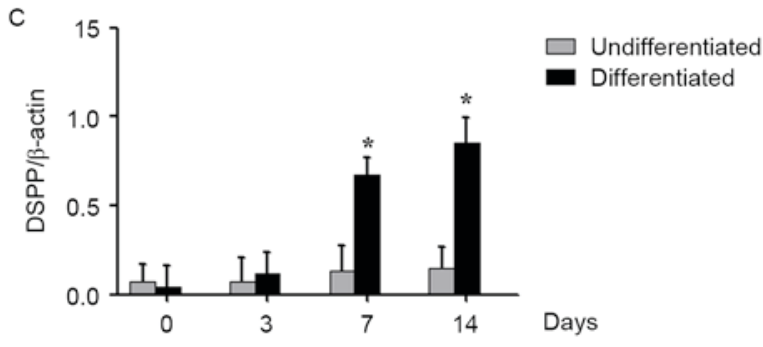

B

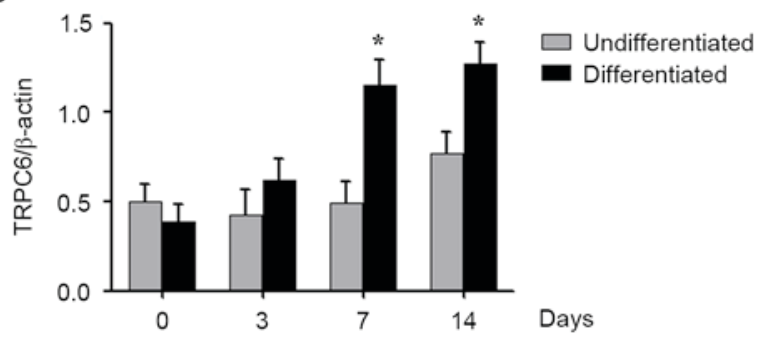

D

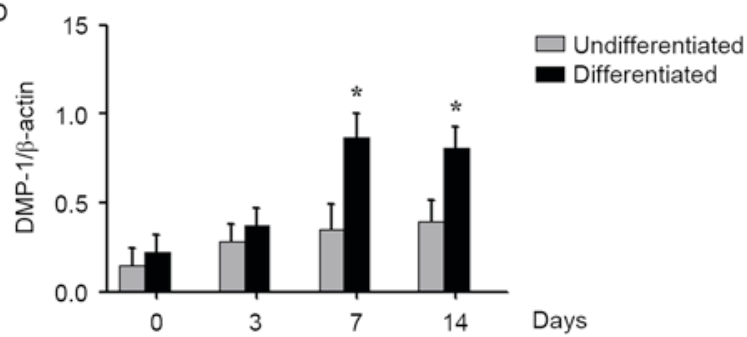

Figure 2. Expression of TRPC6 during odontoblastic differentiation of HDPCs. (A) Western blotting of TRPC6, DSPP, and DMP-1 in HDPCs undergoing odontogenic differentiation, with total proteins collected at 0,3, 7 and 14 days. Quantitative analysis of (B) TRPC6, (C) DSPP and (D) DMP-1 in HDPCs is shown. The results are expressed as the mean \pm standard deviation of three different experiments. ${ }^{*} \mathrm{P}<0.05$ vs. cells on day 0 . HDPCs, human dental pulp cells; TRPC6, transient receptor potential channel 6; DSPP, dentin sialophosphoprotein; DMP-1, dentin matrix protein 1.

downregulation of TRPC6 inhibited the odontoblastic differentiation and mineralization, as indicated by the reduction in the deposition of mineralized matrix and by the downregulation of DSPP and DMP-1 levels. DMP-1 and DSPP are known as mineralization markers in the odontoblasts-like differentiation of HDPCs. DMP-1 is essential to the formation and mineralization of dentine (22), while DSPP is the predominant non-collagen protein in dentin formation $(23,24)$. These results demonstrated a tight coupling between TRPC6 and odontoblastic differentiation, which may be mediated by calcium signaling.
A recent study brought to light that the ORAI1 protein, a calcium channel operated by calcium store, served an important role in odontogenic differentiation (9). Knockdown of ORAI1 expression suppressed odontogenic differentiation and mineralization in vivo and in vitro (9). Meanwhile, it has been confirmed that mutation of ORAI1 may lead to the deficiency of dental enamel calcification (25). TRPC6 is believed to be activated by DAG and it is not sensitive to the change of calcium concentration in the endoplasmic reticulum, which defined TRPC6 as a receptor-operated channel rather than a store-operated channel $(11,26)$. However, several 
A

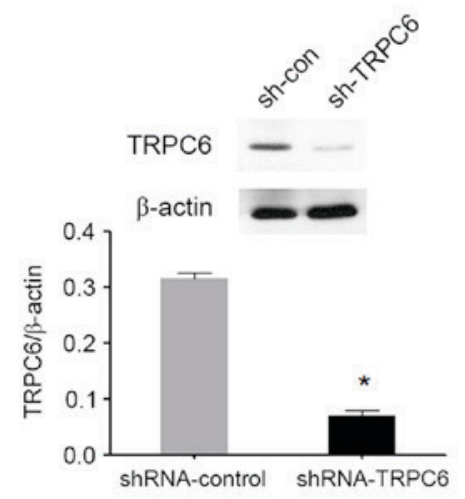

$\mathrm{C}$

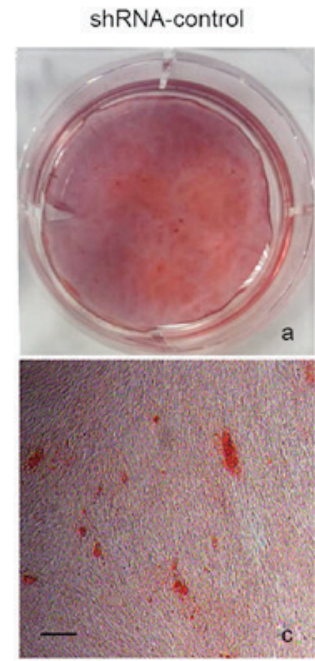

shRNA-TRPC6

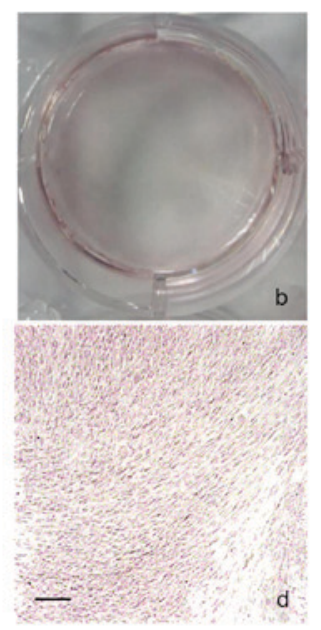

$\mathrm{B}$

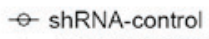

$\triangle$ shRNA-TRPC6

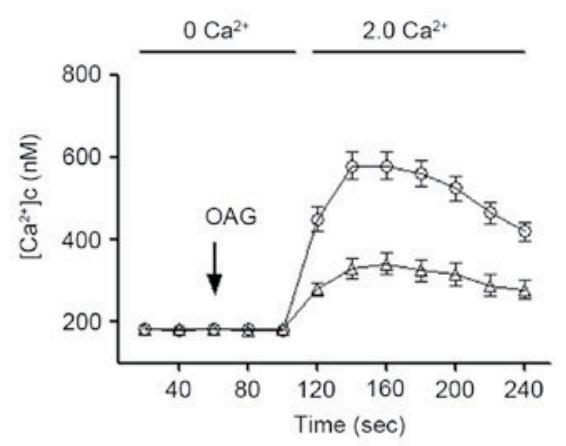

D

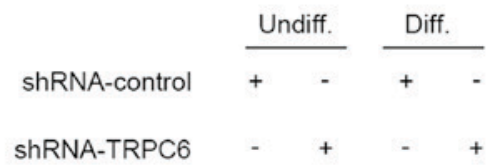

TRPC6

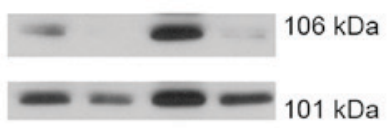

DMP-1

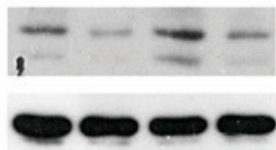

$57 \mathrm{kDa}$

$43 \mathrm{kDa}$
E

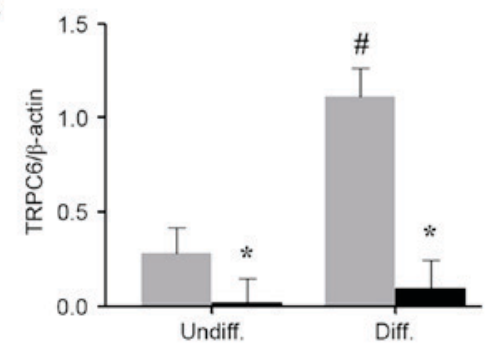

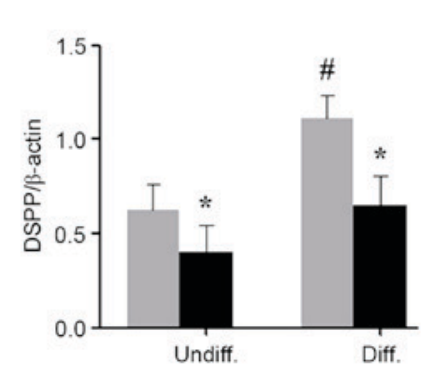

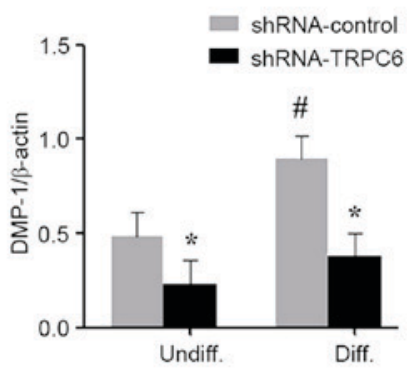

Figure 3. Effect of TRPC6 shRNA on odontoblastic differentiation of HDPCs. (A) Western blotting was performed to confirm the successful TRPC6 knockdown. (B) Intracellular $\mathrm{Ca}^{2+}$ imaging assay was performed to confirm the TRPC6 function following shRNA transfection. (C) Alizarin red S staining in HDPCs on day 14 after odontogenic differentiation in the absence or presence of TRPC6 shRNA. Scale bar, $200 \mu$ m. Magnification, x50. (D) Western blotting was performed to examine the expression levels of TRPC6, DSPP and DMP-1 in all groups. (E) Quantitative analysis of TRPC6, DSPP and DMP-1 protein expression levels in HDPCs. The results are expressed as the mean \pm standard deviation of three different experiments. "P $<0.05$ vs. control group; ${ }^{\# P}<0.05$ vs. undifferentiated control. HDPCs, human dental pulp cells; TRPC6, transient receptor potential channel 6; DSPP, dentin sialophosphoprotein; DMP-1, dentin matrix protein 1; OAG, 1-oleoyl-2-acetyl-sn-glycerol; Undiff, undifferentiated; Diff, differentiated.

lines of evidence indicated that TRPC6 may also affect the SOCE $(27,28)$. Notably, TRPC6 was also found to react with ORAI1 (29). Although the detailed mechanism remains debated, functional studies in different cell types implied a direct link between TRPC6, $\mathrm{Ca}^{2+}$ signaling and cellular responses. Collectively, these factors affected various cellular functions and maintained the cellular homeostasis (18,30-32).

$\mathrm{Ca}^{2+}$ was recognized to be a critical cellular cation that regulates physiological and pathologic processes, including proliferation, transcription and contraction. In clinical practice, $\mathrm{Ca}^{2+}$ released from pulp-capping materials was proven to participate in forming calcium carbonate, which affected the proliferation and differentiation of HDPCs and then promoted the mineralization (33). Furthermore, an in vitro study observed that addition of extracellular $\mathrm{Ca}^{2+}$ increased the expression of bone-associated genes, including BMP-2, and promoted odontoblastic differentiation of dental pulp cells (34). Therefore, further investigations focusing on the association between TRPC6 and calcium signaling, as well as the possible underlying pathway, are required.

In conclusion, the present study demonstrated that TRPC6 was expressed in dental pulp tissue and was involved in the 
odontogenic differentiation process of HDPCs. This raised the possibility that TRPC6 may be a useful therapeutic target in promoting reparative dentin formation.

\section{Acknowledgements}

The present study was supported by grants from the National Natural Science Foundation of China (no. 81600862, 81670984 and 81560184) and the Guangdong Natural Science Foundation (no. 2016A030310227).

\section{References}

1. Aguilar P and Linsuwanont P: Vital pulp therapy in vital permanent teeth with cariously exposed pulp: A systematic review. J Endod 37: 581-587, 2011.

2. Cho SY, Seo DG, Lee SJ, Lee J, Lee SJ and Jung IY: Prognostic factors for clinical outcomes according to time after direct pulp capping. J Endod 39: 327-331, 2013.

3. Mizuno $M$ and Banzai Y: Calcium ion release from calcium hydroxide stimulated fibronectin gene expression in dental pulp cells and the differentiation of dental pulp cells to mineralized tissue forming cells by fibronectin. Int Endod J 41: 933-938, 2008.

4. Natale LC, Rodrigues MC, Xavier TA, Simões A, de Souza DN and Braga RR: Ion release and mechanical properties of calcium silicate and calcium hydroxide materials used for pulp capping. Int Endod J 48: 89-94, 2015.

5. Sangwan P, Sangwan A, Duhan J and Rohilla A: Tertiary dentinogenesis with calcium hydroxide: A review of proposed mechanisms. Int Endod J 46: 3-19, 2013.

6. Barradas AM, Fernandes HA, Groen N, Chai YC, Schrooten J, van de Peppel J, van Leeuwen JP, van Blitterswijk CA and de Boer J: A calcium-induced signaling cascade leading to osteogenic differentiation of human bone marrow-derived mesenchymal stromal cells. Biomaterials 33: 3205-3215, 2012.

7. Berridge MJ, Bootman MD and Roderick HL: Calcium signalling: Dynamics, homeostasis and remodelling. Nat Rev Mol Cell Biol 4: 517-529, 2003.

8. Cheng KT, Ong HL, Liu X and Ambudkar IS: Contribution and regulation of TRPC channels in store-operated $\mathrm{Ca}^{2+}$ entry. Curr Top Membr 71: 149-179, 2013.

9. Sohn S, Park Y, Srikanth S, Arai A, Song M, Yu B, Shin KH, Kang MK, Wang C, Gwack Y, et al: The Role of ORAI1 in the odontogenic differentiation of human dental pulp stem cells. J Dent Res 94: 1560-1567, 2015.

10. Kwon M, Baek SH, Park CK, Chung G and Oh SB: Single-cell RT-PCR and immunocytochemical detection of mechanosensitive transient receptor potential channels in acutely isolated rat odontoblasts. Arch Oral Biol 59: 1266-1271, 2014.

11. Hofmann T, Obukhov AG, Schaefer M, Harteneck C Gudermann T and Schultz G: Direct activation of human TRPC6 and TRPC3 channels by diacylglycerol. Nature 397: 259-263, 1999.

12. Lin Z, Song Z, Qin W, Li J, Li WJ, Zhu HY and Zhang L: Expression of nucleotide-binding oligomerization domain 2 in normal human dental pulp cells and dental pulp tissues. J Endod 35: 838-842, 2009.

13. Qin W, Lin ZM, Deng R, Li DD, Song Z, Tian YG, Wang RF, Ling JQ and Zhu XF: p38a MAPK is involved in BMP-2-induced odontoblastic differentiation of human dental pulp cells. Int Endod J 45: 224-233, 2012.

14. Grynkiewicz G, Poenie M and Tsien RY: A new generation of $\mathrm{Ca}^{2+}$ indicators with greatly improved fluorescence properties. J Biol Chem 260: 3440-3450, 1985.

15. Monet M, Francoeur N and Boulay G: Involvement of phosphoinositide 3-kinase and PTEN protein in mechanism of activation of TRPC6 protein in vascular smooth muscle cells. J Biol Chem 287: 17672-17681, 2012.
16. Chen S, He FF, Wang H, Fang Z, Shao N, Tian XJ, Liu JS, Zhu ZH, Wang YM, Wang S, et al: Calcium entry via TRPC6 mediates albumin overload-induced endoplasmic reticulum stress and apoptosis in podocytes. Cell Calcium 50: 523-529, 2011.

17. Foster RR, Zadeh MA, Welsh GI, Satchell SC, Ye Y Mathieson PW, Bates DO and Saleem MA: Flufenamic acid is a tool for investigating TRPC6-mediated calcium signalling in human conditionally immortalised podocytes and HEK293 cells. Cell Calcium 45: 384-390, 2009

18. Albarran L, Berna-Erro A, Dionisio N, Redondo PC, Lopez E, Lopez JJ, Salido GM, Brull Sabate JM and Rosado JA: TRPC6 participates in the regulation of cytosolic basal calcium concentration in murine resting platelets. Biochim Biophys Acta 1843: 789-796, 2014.

19. Linde A and Lundgren T: From serum to the mineral phase. The role of the odontoblast in calcium transport and mineral formation. Int J Dev Biol 39: 213-222, 1995.

20. Hilkens P, Gervois P, Fanton Y, Vanormelingen J, Martens W, Struys T, Politis C, Lambrichts I and Bronckaers A: Effect of isolation methodology on stem cell properties and multilineage differentiation potential of human dental pulp stem cells. Cell Tissue Res 353: 65-78, 2013.

21. Kawashima N: Characterisation of dental pulp stem cells: A new horizon for tissue regeneration? Arch Oral Biol 57: 1439-1458, 2012.

22. Butler WT and Ritchie $\mathrm{H}$ : The nature and functional significance of dentin extracellular matrix proteins. Int J Dev Biol 39: 169-179, 1995.

23. Lee SY, Kim SY, Park SH, Kim JJ, Jang JH and Kim EC: Effects of recombinant dentin sialoprotein in dental pulp cells. J Dent Res 91: 407-412, 2012.

24. Lee YH, Kim GE, Cho HJ, Yu MK, Bhattarai G, Lee NH and Yi HK: Aging of in vitro pulp illustrates change of inflammation and dentinogenesis. J Endod 39: 340-345, 2013.

25. McCarl C, Picard C, Khalil S, Kawasaki T, Röther J, Papolos A, Kutok J, Hivroz C, Ledeist F, Plogmann K, et al: ORAI1 deficiency and lack of store-operated $\mathrm{Ca}^{2+}$ entry cause immunodeficiency, myopathy, and ectodermal dysplasia. J Allergy Clin Immunol 124: 1311-1318. e7, 2009.

26. Parekh AB and Putney JW Jr: Store-operated calcium channels. Physiol Rev 85: 757-810, 2005.

27. Ichikawa J and Inoue R: TRPC6 regulates cell cycle progression by modulating membrane potential in bone marrow stromal cells. Br J Pharmacol 171: 5280-5294, 2014.

28. El Boustany C, Bidaux G, Enfissi A, Delcourt P, Prevarskaya N and Capiod T: Capacitative calcium entry and transient receptor potential canonical 6 expression control human hepatoma cell proliferation. Hepatology 47: 2068-2077, 2008.

29. Jardin I, Gómez LJ, Salido GM and Rosado JA: Dynamic interaction of hTRPC6 with the Orail-STIM1 complex or hTRPC3 mediates its role in capacitative or non-capacitative $\mathrm{Ca}(2+)$ entry pathways. Biochem J 420: 267-276, 2009.

30. Winn MP, Conlon PJ, Lynn KL, Farrington MK, Creazzo T, Hawkins AF, Daskalak is N, Kwan SY, Ebersviller S, Burchette JL, et al: A mutation in the TRPC6 cation channel causes familial focal segmental glomerulosclerosis. Science 308: 1801-1804, 2005.

31. Ding Y, Winters A, Ding M, Graham S, Akopova I, Muallem S, Wang Y, Hong JH, Gryczynski Z, Yang SH, et al: Reactive oxygen species-mediated trpc6 protein activation in vascular myocytes, a mechanism for vasoconstrictor-regulated vascular tone. J Biol Chem 286: 31799-31809, 2011.

32. Tauseef M, Knezevic N, Chava KR, Smith M, Sukriti S, Gianaris N, Obukhov AG, Vogel SM, Schraufnagel DE, Dietrich A, et al: TLR4 activation of TRPC6-dependent calcium signaling mediates endotoxin-induced lung vascular permeability and inflammation. J Exp Med 209: 1953-1968, 2012.

33. An S, Gao Y, Ling J, Wei X and Xiao Y: Calcium ions promote osteogenic differentiation and mineralization of human dental pulp cells: Implications for pulp capping materials. J Mater Sci Mater Med 23: 789-795, 2012.

34. Tada H, Nemoto E, Kanaya S, Hamaji N, Sato H and Shimauchi H: Elevated extracellular calcium increases expression of bone morphogenetic protein-2 gene via a calcium channel and ERK pathway in human dental pulp cells. Biochem Biophys Res Commun 394: 1093-1097, 2010. 